\title{
Economic analysis and optimization of energy technology based on the matrix model thermoeconomics theory
}

\author{
Liang Chen \\ School of Economics and Trade, Hunan University, Changsha 410082, China
}

Corresponding Author Email: Chliang000@163.com

https://doi.org/10.18280/ijht.360410

Received: 14 January 2018

Accepted: 23 July 2018

\section{Keywords:}

thermoeconomics, matrix model, energy technology, economic analysis

\begin{abstract}
The matrix model thermoeconomics theory has been widely applied in the energy technology analysis to optimize energy technologies, improve energy utilization efficiency and reduce pollutant emissions. This paper uses the matrix model thermoeconomics theory to analyze the thermodynamics and economics of the energy thermodynamic system, adopts the simulation technology for modelling and then gives an optimized strategy. According to the research results, the matrix model thermoeconomics theory takes into account both thermodynamics and economics when being applied to address energy technology problems, so the analysis is more comprehensive and accurate; the improved local-global decomposition optimization method (LGDO), by combining the advantages of both "global optimization" and "local optimization", has the characteristics of fast convergence and low error, making it more applicable. Through this research, it can be found that the energy technology analysis based on the matrix model thermoeconomics theory is of important theoretical and practical significance in improving the energy utilization and core competitiveness of energy technologies in China.
\end{abstract}

\section{INTRODUCTION}

Energy is an important guarantee for the rapid economic and social development. The effective use of energy is the core of energy engineering technologies. Since the reform and opening up, more and more energy has been consumed, and at the same time, environmental pollution and global warming have gradually raised people's concern. "Energy conservation and emission reduction" is the theme of the current era and also the development direction of energy technologies in China. Thermodynamic analysis relies mainly on the first law and the second law of thermodynamics, which can only locally analyze system efficiency and quantify the rate of energy consumption. Economic analysis can calculate the fuel consumption as well as the operating and maintenance costs of the system as a whole, but cannot evaluate the cost distribution of local subsystems [1]. Therefore, thermodynamics and economics can be combined with each other to make the analysis more comprehensive. Economics and thermodynamics share similarities in some concepts. In economics, "cost" is featured with "scarcity" and "loss", which is similar to the concept of "exergy" in thermodynamics. In this way, a multi-disciplinary analysis method is formed the thermoeconomics theory [2]. After decades of research and development, this theory has been gradually expanded, from which, the matrix model thermoeconomics theory has emerged and is now being more and more widely accepted. It adopts the general mathematical structure of a linear model, and involves cost accounting and energy efficiency [3]. The thermoeconomics theory can be used not only to perform cost analysis of a system to improve energy efficiency, but also to reduce energy consumption cost.

According to the existing literatures, the thermoeconomics theory consists of two parts - cost analysis and system optimization. The cost analysis method based on thermoeconomics mainly applies cost accounting, which is used to estimate the total cost of each product or energy input, including the capital expenditures (capex) and the operating expenditures (opex) [4]. At first, in the analysis of complex system costs, concepts like "extraction" and "equivalence" were used in the construction of auxiliary equations [5]. Scholars then established and developed the theory of exergy cost, which laid the foundation for the subsequent development of thermoeconomics. At that time, the hypothesesand inferences about fuel, energy, and product were successively proposed and used as the basis for the construction of auxiliary equations [6]. Based on the auxiliary equations and the above hypotheses, it is concluded that, once the optimization problem is determined, the cost defined in the exergy cost theory is consistent with the Lagrangian multiplier; in other words, it is consistent with the optimization theory of marginal cost [7]. In the thermoeconomic system optimization field, an optimization problem mainly attempts to figure out which strategies and configurations can be used to make the whole system reach the optimum under the established constraints and conditions [8]. The "onion model" and "pinch analysis" methods were first used in the optimization of complex energy systems. Despite many problems there, they did open the door to the optimization of thermoeconomic systems [9]. With the analysis technology of the second law of thermodynamics established, the complex energy system optimization model has been improved. Entropy generation minimization is the most commonly used method, which combines the conventional thermodynamic analysis with the heat transfer media to clearly identify the principle of entropy generation [10]. However, the optimization method based on 
the second law of thermodynamics cannot identify and evaluate the system from the global perspective, and also shows some drawbacks in balancing the global system performance and optimizing the investment cost [11]. The establishment of modern thermoeconomics makes up for these drawbacks. It connects the investment cost of each sub-device together and takes the total cost of the system as the objective function, which can reflect the thermodynamic and economic performance of the system [12]. Scholars then improved the conventional energy-mass analysis method and combined it with the second law of thermodynamics to create the "thermoeconomic functional analysis" method [13], and at the same time proved the consistency between the thermoeconomic analysis method and the conventional thermodynamic analysis method, which paved the way for the application of thermodynamic analysis in the system optimization. This method can achieve convergence fast and well handle the optimization of complex energy systems [14].

Through the review and analysis of the above literatures, it is found that the current research is mostly carried out from two aspects - cost analysis and system optimization. The overall system analysis is not comprehensive, the parameter settings complicated and the calculation process extremely complex, often making it impossible to obtain the optimal solution. In order to overcome this shortcoming, the matrix model thermoeconomics analysis method came into being, which divides the energy production system into several interrelated subsystems, and then obtains the cost balance matrix equations according to the subsystems. With the necessary supplementary equations, the total product cost is finally obtained, which can objectively and accurately reflect the substantial loss of energy. This method is rarely used in the current literatures, except in transportation facilities such as aircraft and ship fuel consumption [15]. There is no economic analysis on the core of the energy technology applications (such as the energy efficiency of the power plant). This paper uses the matrix model thermoeconomics theory to conduct economic analysis and optimization of the coal-fired power generation systems. In theory, it enriches and develops the research methods for thermoeconomics and energy economics; and in reality, it improves energy efficiency and reduces the discharge of pollutants and greenhouse gases, which has important practical significance to the development of green economy and circular economy.

\section{ESTABLISHMENT AND SOLUTION OF THE SIMULATION MODEL FOR ENERGY THERMODYNAMIC SYSTEM AND CARBON DIOXIDE EMISSION REDUCTION SYSTEM}

\subsection{Establishment of the simulation model for the energy thermodynamic system}

The system simulation technology is widely used in thermoeconomics analysis due to its reproducibility, safety and high efficiency. The coal-fired thermal system is very large, with an extremely complicated equipment composition, so it should be simulated with both linear and nonlinear models. To simulate a complex coal-fired system, the theoretical model is usually applied in combination with the black box model. Figure 1 shows the simulation model of the thermodynamic system of the power plant. According to the operating characteristics, the equipment is divided into four types: heat exchange, transmission, expansion and compression. For each piece of equipment, three mathematical equations need to be established, namely the balance of mass, energy and heat.

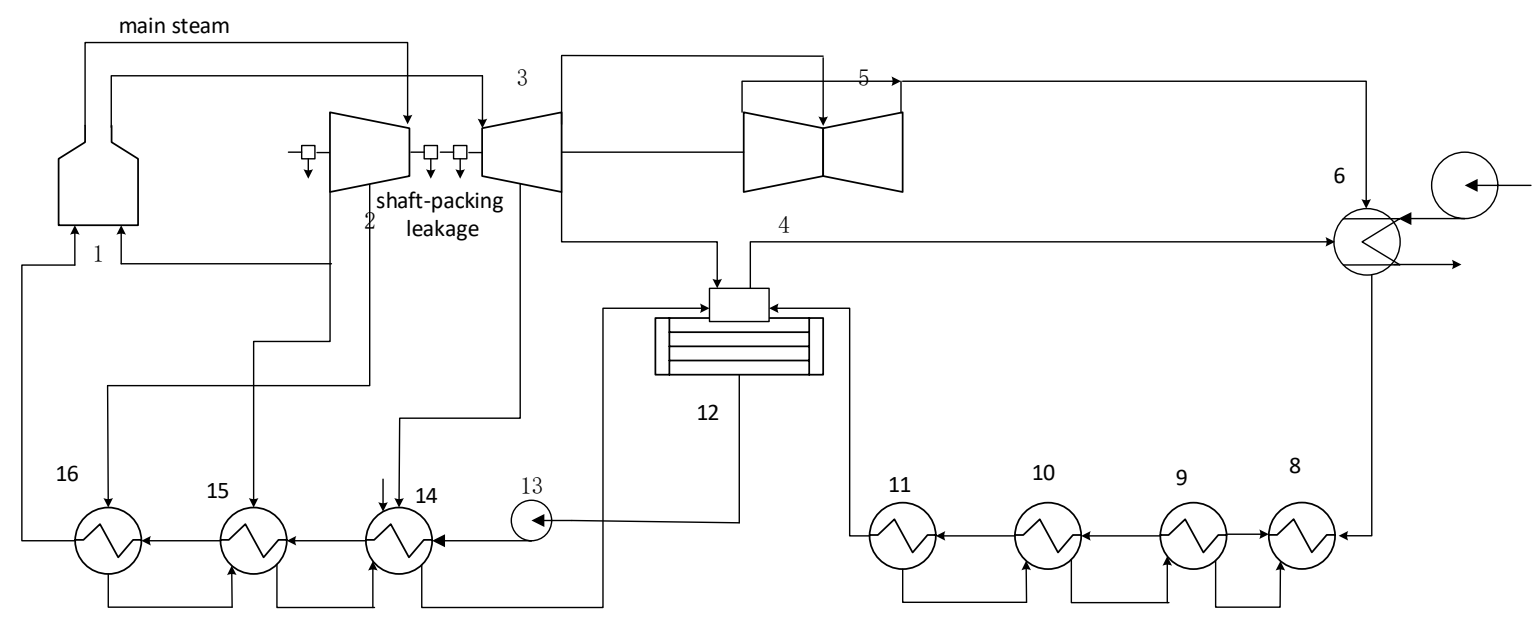

Figure 1. Schematic diagram of the simulated thermodynamic system

\subsection{Establishment of the simulation model for the carbon dioxide emission reduction system}

With the advanced carbon dioxide isolation technology, this paper builds a simulation model based on cyclic combustion and MEA adsorption, as shown in Figure 2. These two types of emission reduction systems have less impact on the structure of a conventional power plant, so this model is simplified. During the operation of the system, the sour gas reacts with MEA to form thermally stable salt, and $\mathrm{SO}_{2}$ is absorbed by MEA and recycled by heating and distillation. 


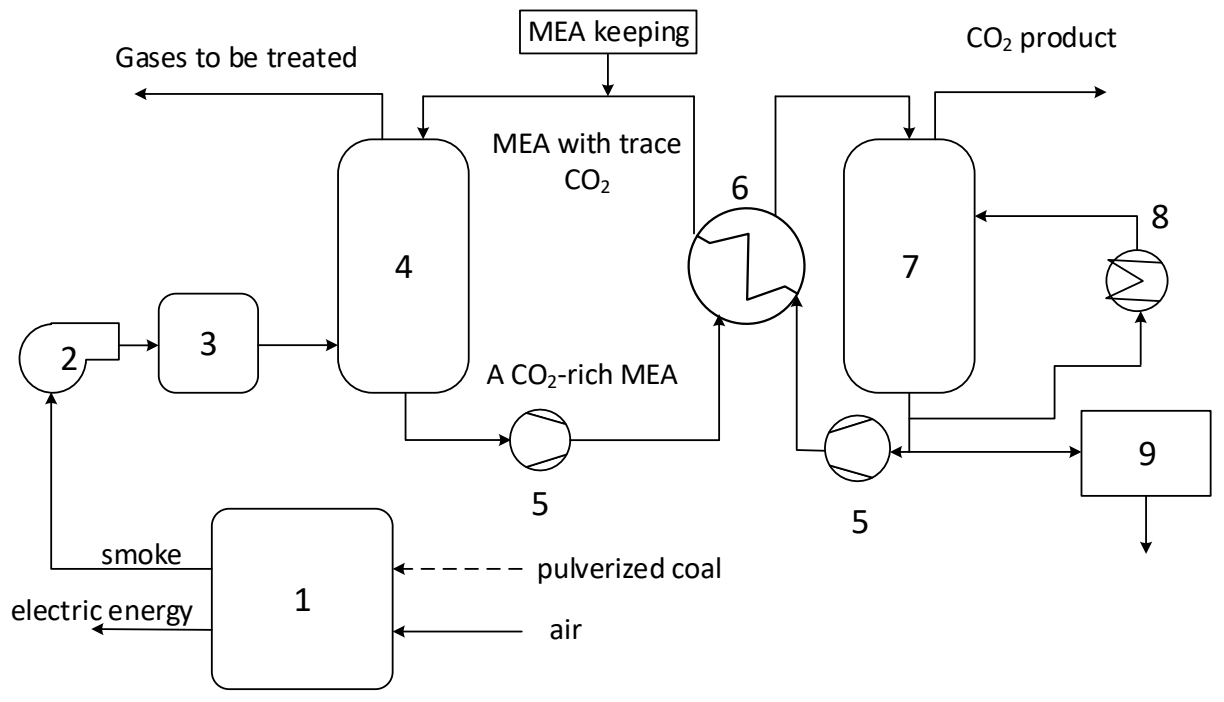

Figure 2. Simplified diagram of adsorption system simulation

\subsection{Solution to the energy thermodynamic system algorithm}

According to the thermodynamic steady-state model and the second law of thermodynamics, combined with the mass and energy balance equations and the thermodynamic characteristic equation, the mathematical model for the thermodynamic system of the coal-fired power plant is obtained, which is a linear and nonlinear mixed equation set with 60 parameter variables. This paper uses the Powell hybrid method to solve it. The tolerance for global convergence is set to be $10^{-6}$, and the algorithm flow of the system equation solution is shown in Figure 3.

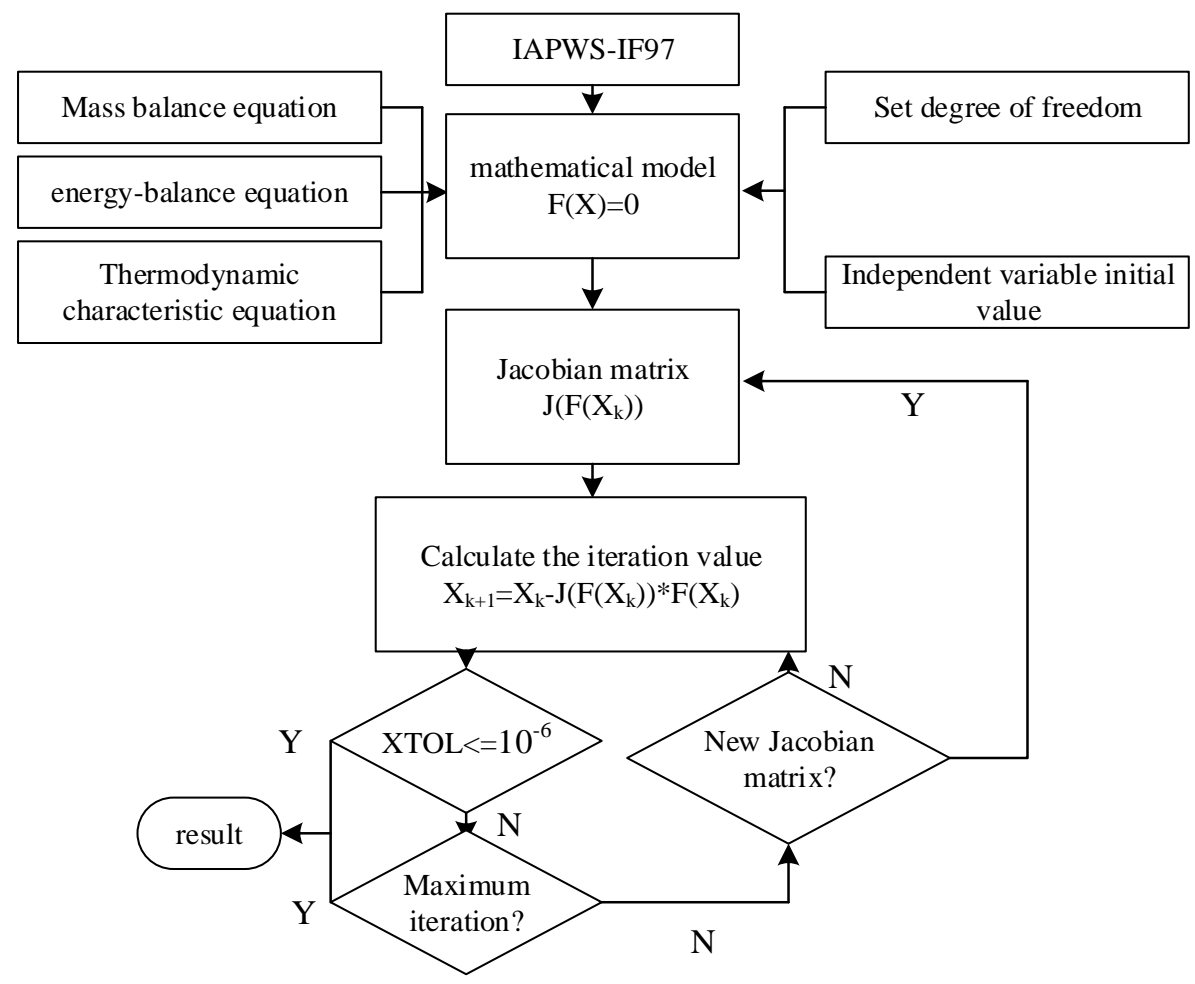

Figure 3. Algorithm flow chart of the system equation solution

\section{THERMOECONOMIC COST ANALYSIS OF THE ENERGY THERMODYNAMIC SYSTEM}

Section 2 of this paper is based on the simulation technology and the thermodynamic analysis method. In order to better analyze the energy technology, this paper adopts the matrix model thermoeconomics theory, which puts the system into two environments for observation. The first one is the natural environment, also called the physical environment, where the parameter indicators are the physical variables of thermodynamics, such as temperature, heat and pressure, etc., which obey the first and second laws of thermodynamics and are bound by many laws of nature; the other one is the economic environment, where the variables are price, cost, profit, supply and demand, etc., which are also subject to a series of economic laws. The thermoeconomics model 
combining the two can meet the balance of mass, energy and cost.

\subsection{Establishment of the thermoeconomics model for the energy thermodynamic system}

The thermoeconomics model is constructed by combining the economic costs of equipment and raw materials with the laws of thermodynamics. In order to comprehensively and accurately analyze the overall cost of the energy thermodynamic system, the components in the system are divided into fuel flow and product flow. The former provides energy to the entire system, and the product gets energy and gains "added value". The difference between the fuel inflow and outflow is the total fuel $(\mathrm{F})$ required for the operation of the entire system; the difference between the product outflow and inflow is the effective energy transferred, and also the part that realizes "added value", i.e. the product $(\mathrm{P})$; and the difference between the total product and total energy is exergy loss. In general, the losses of the whole system are summarized as follows: (1) exergy consumed by regenerative extraction (FB $\left.=B_{2}+B_{4}-B_{5}\right)$; (2) exergy consumed by water supply equipment ( $\left.\mathrm{P}=B_{3}-B_{1}\right)$; (3) one is the exergy consumed by the gas/water circulation ( $\left.\mathrm{FB}=B_{1}+B_{2}+B_{4}-B_{3}\right)$; and the other is the energy consumed by the water circulation of the pump ( $\mathrm{FW}=\mathrm{W}$ ). According to the thermodynamic irreversible entropy production principle, the fuel resources additionally consumed (FS) and the unit exergy consumed by each piece of equipment are calculated using the formulas as shown in Table 1.

Table 1. System unit loss equations

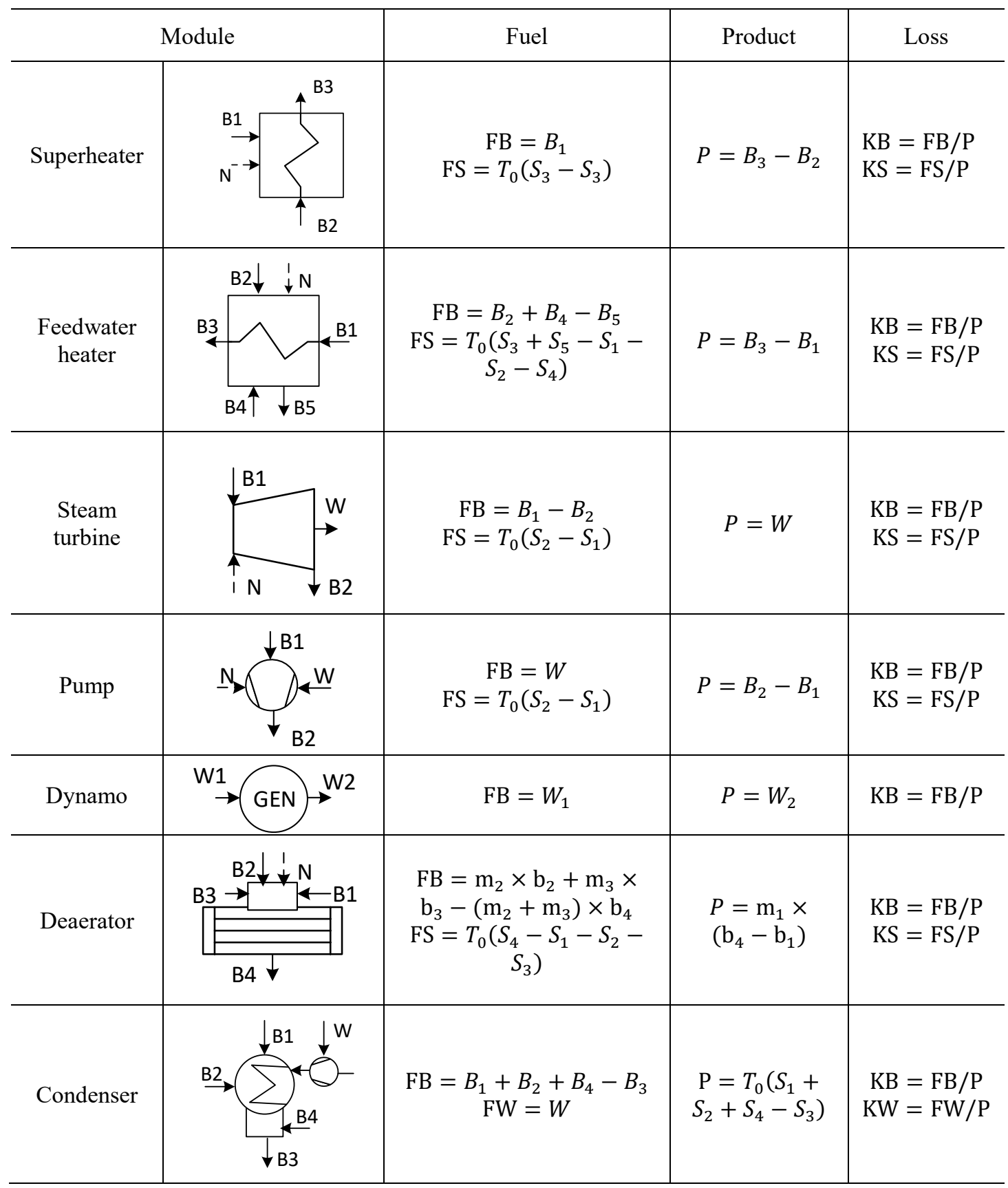


3.2 Solution of the energy thermodynamic system model and the thermodynamic and economic calculation

According to the previous energy thermodynamic system model, the economic cost equations of the various equipment of the system are solved simultaneously to obtain the average thermoeconomic cost of the product. This paper mainly analyzes the effect of the main steam $\mathrm{T}_{\mathrm{SH}}$ (superheated steam and reheated steam temperature) on the system stability,

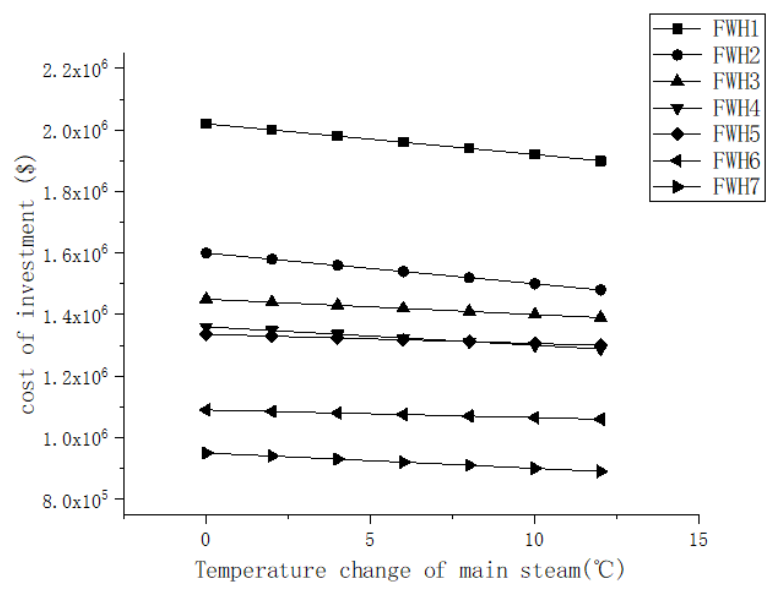

Figure 4. FWH investment cost curves

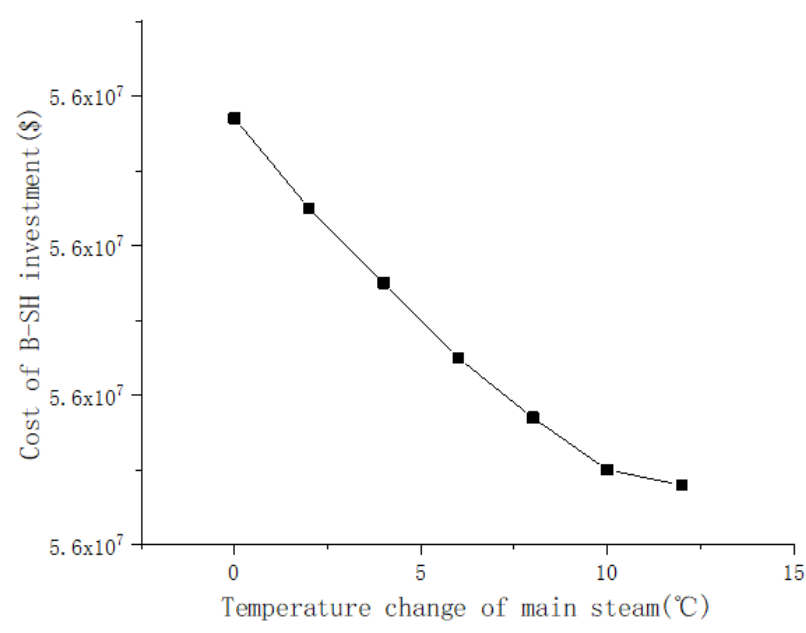

Figure 6. B-SH investment cost curve

From Figure 4-7, it can be seen that with the investment cost of the component FWH tends to decrease slowly with the increase of the main steam temperature; for HP1, LP1 and LP2, the investment cost increases with the increase of temperature, and the magnitude of the increase is getting larger; and for B$\mathrm{SH}$, the investment cost decreased to some extent with the increasing temperature. On the whole, under the given system load, with the increase of the main steam temperature, the annual coal-fired cost decreases, but the annual investment cost shows an increasing trend. When both are taken into account, the annual total cost declines first and then rise.

\section{THERMOECONOMIC OPTIMIZATION OF THE ENERGY THERMODYNAMIC SYSTEM}

The optimization of an energy system can save fuels while reducing greenhouse gas emissions. The optimization methods operation efficiency and power generation cost in the energy thermodynamic system. In the model analysis, the total system load remains constant and the temperature is controlled between $3^{\circ} \mathrm{C}$ and $13^{\circ} \mathrm{C}$. According to the above settings, the investment cost of each system component (FWH1-FWH7, HP1, LP1 and LP2 components) is calculated, and the corresponding variation curves are shown in Figures 4, 5, 6 and 7.

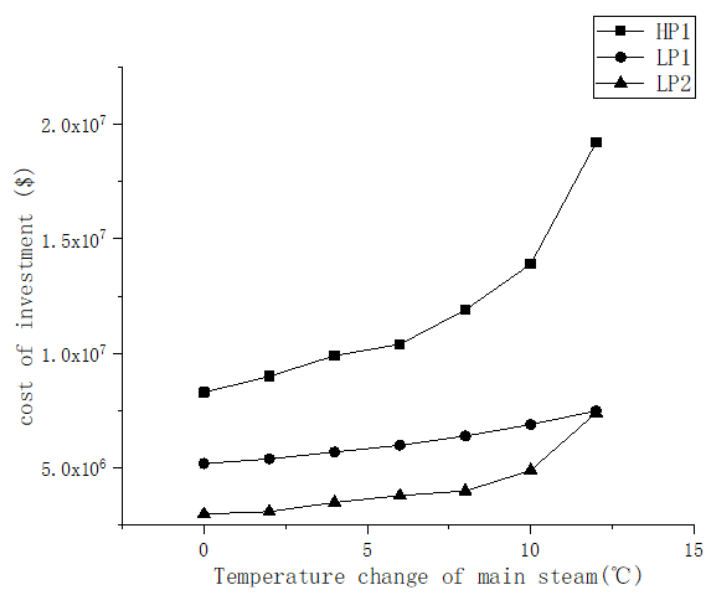

Figure 5. HP1, LP1 and LP2 investment cost curves

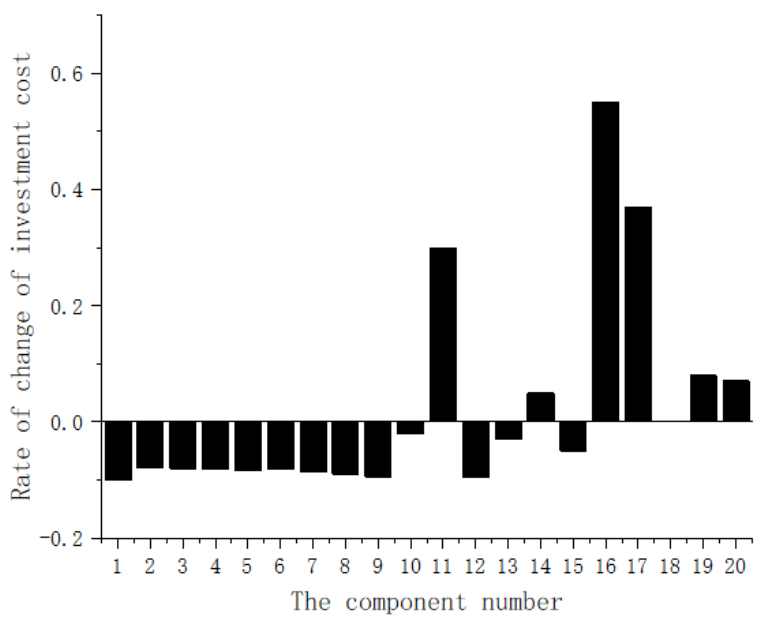

Figure 7. Histogram of the change rate of investment cost

for thermoeconomics include local optimization, global optimization and local-global decomposition optimization. The local optimization method can achieve convergence quickly, but the results are often inaccurate, and there are complex couplings between subsystems, which usually makes it unable to achieve global optimization. The global optimization method has high precision and can achieve global optimization, but the operation is extremely complicated. Under the existing computing capacity, the convergence is very slow, with huge calculation amount, and the existing mathematical algorithm is not mature enough to be applied in practice, because in reality, the optimization involves too many variables and cannot obtain a convergent solution. The local-global decomposition optimization method combines the advantages of both local and global optimization methods and takes into account both accuracy and computational complexity. This paper proposes an improved local-global decomposition optimization method (LGDO) based on the 
existing one and adopts the above model to optimize the energy system. The system decomposition strategy adopted in this paper can minimize the couplings between subsystems so that the decomposable subsystems apply local optimization, while the non-decomposable ones global optimization. In this way, the overall optimization can infinitely approximate the global optimization in the true sense. For the 20 feature variables selected in the previous section, each of them may not affect the components of the system to which it belongs only. When the independent variable $\mathrm{x}$ affects $\mathrm{m}$ components, the optimization objective function is the sum of the thermoeconomic cost of the product:

$$
\begin{aligned}
& \min _{x} \Gamma=\sum_{j=1}^{m} c_{p, j} P_{j}=\sum_{j=1}^{m}\left(\sum_{i=0}^{n} k_{i j}(x, y) c_{p, i}+\right. \\
& \left.k Z_{j}(x, y)\right) P_{j}
\end{aligned}
$$

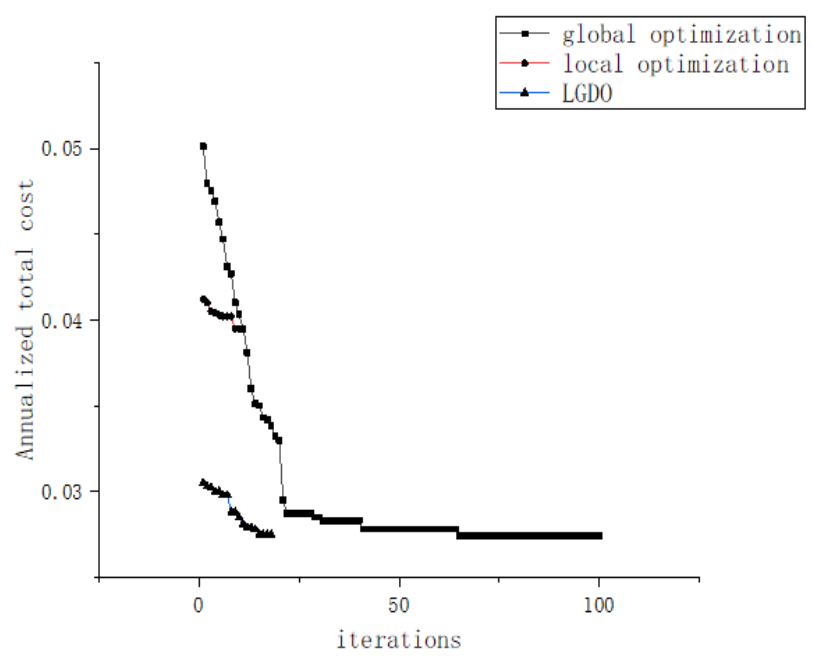

Figure 8. Comparison of iterative convergence of three optimization methods

\section{CONCLUSIONS}

This paper uses the matrix model thermoeconomics theory to analyze an energy technology for the purpose of improving the energy utilization rate and reducing the discharge of pollutants. Through the matrix model method combining thermodynamics and economics, simulation and modelling and the local-global decomposition optimization method (LGDO), the following conclusions are obtained:

(1) The matrix model thermoeconomic analysis method combines the thermodynamic and economic analysis. With the aid of the simulation technology, this paper conducts analysis and optimization under the laws of thermodynamics and the rules of economic operations, and the results obtained are consistent with the actual situation.

(2) The improved LGDO method is featured with fast convergence speed and low error, making it better than the conventional "global optimization" and "local optimization" methods.

(3) Through the instance analysis of an energy thermodynamic system, it is found that the total system cost declines first and then rise. After LGDO is applied, the thermoeconomic costs of most system components are significantly reduced.
The conventional local optimization and global optimization methods are here compared with the improved LGDO method in terms of the convergence speed and relative error. As shown in Fig. 8, the error of the optimal solution obtained by the LGDO method is very small, and the convergence speed is quite fast, while the local optimization has fast convergence speed, but the error is large, and the global optimization method results in a small error but the convergence takes far more time than LGDO and the local optimization method. Judging from the optimization results of LGDO, the thermoeconomic costs of most components are significantly reduced (Figure 9).

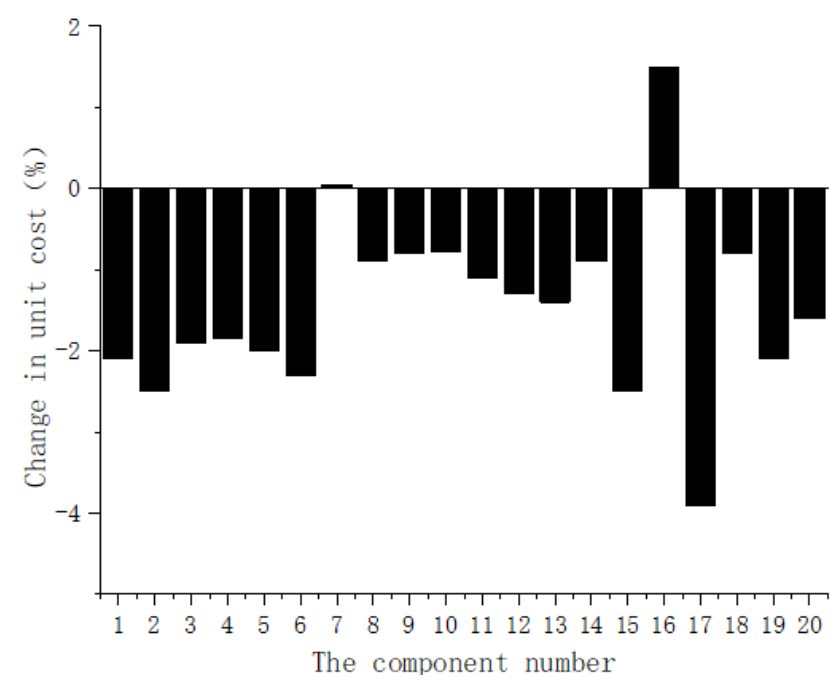

Figure 9. Rate of change of the component cost before and after optimization

\section{REFERENCES}

[1] El-Sayed YM. (1999). Thermoeconomics of some options of large mechanical vapor-compression units. Desalination 125(1-3): 251-257. https://doi.org/10.1016/s0011-9164(99)00146-0

[2] Usón S, Valero A, Agudelo A. (2012). Thermoeconomics and industrial symbiosis. effect of byproduct integration in cost assessment. Energy 45(1): 4351. https://doi.org/10.1016/j.energy.2012.04.016

[3] Cardona E, Piacentino A. (2007). Optimal design of chcp plants in the civil sector by thermoeconomics. Applied Energy $\quad 84(7)$ : 729-748. https://doi.org/10.1016/j.apenergy.2007.01.005

[4] Prestipino M, Chiodo V, Maisano S, Brusca S, Urbani F, Galvagno A. (2018). Hydrogen production from residual biomass via air-steam gasification for a bioenergy-based economy in Sicily. Annales de Chimie - Science des Matériaux 42(4): 441-452. http://dx.doi.org/10.3166/ACSM.42.441-452

[5] Banerjee A, Tierney MJ, Thorpe RN. (2012). Thermoeconomics, cost benefit analysis, and a novel way of dealing with revenue generating dissipative units applied to candidate decentralised energy systems for indian rural villages. Energy 43(1): 477-488. https://doi.org/10.1016/j.energy.2012.03.002 
[6] Sahu MK. (2016). Investigation of the effect of air film blade cooling on thermoeconomics of gas turbine based power plant cycle. Energy 115: 1320-1330. https://doi.org/10.1016/j.energy.2016.09.069

[7] Deng J, Wang R, Wu J, Han G, Wu D, Li S. (2008). Exergy cost analysis of a micro-trigeneration system based on the structural theory of thermoeconomics. Energy 33(9): 1417-1426. https://doi.org/10.1016/j.energy.2008.05.001

[8] Piacentino A. (2015). Application of advanced thermodynamics, thermoeconomics and exergy costing to a multiple effect distillation plant: In-depth analysis of cost formation process. Desalination 371: 88-103. https://doi.org/10.1016/j.desal.2015.06.008

[9] Kwak HY, Kim DJ, Jeon JS. (2003). Exergetic and thermoeconomic analyses of power plants. Energy 28(4): 343-360. https://doi.org/10.1016/s0360-5442(02)00138$\mathrm{x}$

[10] Nafey AS, Fath HES, Mabrouk AA. (2008). Thermoeconomic design of a multi-effect evaporation mechanical vapor compression (mee-mvc) desalination process. Desalination 230(1):

$1-15$. https://doi.org/10.1016/j.desal.2007.08.021

[11] Frangopoulos CA. (1987). Thermo-economic functional analysis and optimization. Energy 12(7): 563-571. https://doi.org/10.1016/0360-5442(87)90097-1

[12] Lian ZT, Chua KJ, Chou SK. (2010). A thermoeconomic analysis of biomass energy for trigeneration. Applied Energy 87(1):

84-95. https://doi.org/10.1016/j.apenergy.2009.07.003

[13] Mabrouk AA, Nafey AS, Fath HES. (2007). Thermoeconomic analysis of some existing desalination processes. Desalination 205(1): 354-373. https://doi.org/10.1016/j.desal.2006.02.059

[14] Hamed OA, Al-Washmi HA, Al-Otaibi HA. (2006). Thermoeconomic analysis of a power/water cogeneration plant. Energy 31(14): 2699-2709. https://doi.org/10.1016/j.energy.2005.12.011

[15] Sayyaadi H, Saffari A. (2010). Thermoeconomic optimization of multi effect distillation desalination systems. Applied Energy 87(4): 1122-1133. 